\title{
Development of Rifapentine Susceptibility Tests for Mycobacterium tuberculosis
}

\author{
L. HEIFETS, ${ }^{*}$ T. SANCHEZ, J. VANDERKOLK, AND V. PHAM \\ National Jewish Medical and Research Center and University of Colorado Health Sciences Center, Denver, Colorado
}

Received 15 June 1998/Returned for modification 29 September 1998/Accepted 25 October 1998

\begin{abstract}
Two methods for testing the susceptibility of Mycobacterium tuberculosis to rifapentine have been developed: the agar proportion method and the radiometric BACTEC technique. A critical concentration of $0.5 \mu \mathrm{g}$ of rifapentine per $\mathrm{ml}$ is proposed for both methods since it provides a reliable means of distinguishing between susceptible and resistant $M$. tuberculosis isolates. It is recommended that two quality control $M$. tuberculosis strains be used at the introduction of these tests in a clinical laboratory: one that is pansusceptible (H37Rv) and one that is resistant to rifapentine. The resistant strain can be obtained from the American Type Culture Collection, where it is deposited under the number ATCC 700457.
\end{abstract}

Rifapentine (RPT) is a long-lasting rifamycin recently approved by the Food and Drug Administration as an alternative to rifampin (RMP) in treatment regimens for patients with tuberculosis. There were many reports on the antimicrobial activity of RPT in vitro $(1,4,7,12,13)$, in macrophages $(3,9$, $10)$, and in mice $(1,2,8,11)$. The purpose of this study was to develop for clinical laboratories a procedure(s) suitable for testing the susceptibility of Mycobacterium tuberculosis isolates to RPT rather than a procedure that again ascertains the well-known data on the activity of this drug against $M$. tuberculosis in vitro. The RPT susceptibility tests were developed for two types of culture media: Middlebrook-Cohn 7H10 and $7 \mathrm{H} 11$ agars and BACTEC 7H12 broth.

\section{MATERIALS AND METHODS}

Laboratory strains. Eight pansusceptible M. tuberculosis strains from our collection were used for preliminary studies with RPT. In addition, RPT-resistant mutants were developed from three of these strains (H37Rv, Erdman, and Atencio).

QC strains. Two quality control (OC) strains were used in experiments with clinical isolates: one pansusceptible strain, M. tuberculosis H37Rv (ATCC 27294), and one strain that had monoresistance to RPT and that was developed in our laboratory (H37RPT-R; see below). The latter strain has been deposited at the American Type Culture Collection (ATCC) as strain ATCC 700457.

Clinical isolates. A total of 95 pansusceptible $M$. tuberculosis isolates from new patients who had not received any prior antituberculosis treatment were included in this study. These isolates were retrieved from cultures of specimens from 52 patients. The 33 RMP-resistant isolates were selected from isolates submitted to our laboratory for routine drug susceptibility testing. Most of these isolates were resistant to two or more drugs, but four of them had monoresistance to RMP.

Drugs. RPT was obtained from Hoechst Marion Roussel, Inc. (Kansas City, Mo.), and RMP was obtained from Sigma Chemical Co. (St. Louis, Mo.). The stock solutions were made in methanol and were subsequently diluted in distilled water before incorporation into culture media.

Media. Two commercially available media were used: (i) $7 \mathrm{H} 12$ broth in $12 \mathrm{~B}$ vials (Becton-Dickinson, Sparks, Md.) and (ii) 7H10 and 7H11 agars made from Middlebrook-Cohn 7H10 agar base (Becton-Dickinson) with 10\% oleic acidalbumin-dextrose-citrate (Remel, Lenexa, Kans.).

The agar medium was distributed in quadrant plastic plates $(5 \mathrm{ml}$ per quadrant) so that three quadrants contained three different drug concentrations and one quadrant in each plate was drug-free.

MIC determination. On the basis of the definition of the MIC as the lowest drug concentration that inhibited more than $99 \%$ of the bacteria in a culture, the MIC on agar plates was determined by comparing the number of colonies in the drug-free control sections of the plate with the number of colonies growing in the presence of drug concentrations ranging from 0.03 to $8.0 \mu \mathrm{g} / \mathrm{ml}$.

To determine the MIC in BACTEC $7 \mathrm{H} 12$ broth, a series of $12 \mathrm{~B}$ vials were

* Corresponding author. Mailing address: National Jewish Medical and Research Center, 1400 Jackson St., Denver, CO 80206. Phone: (303) 398-1384. Fax: (303) 398-1953. E-mail: heifetsl@njc.org. supplemented with RPT or RMP ( 0.03 to $8.0 \mu \mathrm{g} / \mathrm{ml})$, each of which was added in a volume of $0.1 \mathrm{ml}$. A culture from a seed vial, in which the radiometric growth index (GI) reached 999, was diluted 1:10 to inoculate $0.1 \mathrm{ml}$ of it into all drug-containing vials and one of two control vials. Cultures that reached a GI of 500 were used undiluted. An alternative is to use as the inoculum cultures that are grown in 7H9 broth, adjusted to the optical density of a McFarland no. 1 standard, and diluted 1:100. This approach provided an inoculum in the range of $10^{4}$ to $10^{5} \mathrm{CFU} / \mathrm{ml}$. One of two control vials was inoculated with a 1:100 dilution of the bacterial suspension, providing initial contents of $10^{2}$ to $10^{3} \mathrm{CFU} / \mathrm{m}$ (1:100 control). The MIC determined radiometrically in $7 \mathrm{H} 12$ broth was defined as the lowest drug concentration in the presence of which the daily GI did not exceed 50 while in the 1:100 control the daily GI was above the GI in the drug-containing vial for a minimum of 3 consecutive days. The validity of an experiment was controlled by the intensity of growth in the drug-free control vial inoculated with the original suspension used for the drug-containing vials (undiluted control). If the inoculum was in the desired range of $10^{4}$ to $10^{5} \mathrm{CFU} / \mathrm{ml}$, the GI in this control should not be at the maximum GI (GI999) within less than 3 days of cultivation, but the growth should reach a GI of 500 or greater within a maximum of 10 days of cultivation.

Although this BACTEC technology for determining more than $99 \%$ growth inhibition is now a standard procedure, including for RMP susceptibility testing, we felt it necessary to validate the GI radiometric assessment of growth inhibition by performing actual viable counts in experiments with RPT and to use RMP for comparison. More details on MIC determinations in BACTEC broth were given in our previous publications $(5,6)$.

\section{RESULTS}

Validation of the radiometrically determined MIC of RPT. To validate the radiometrically determined MICs of RPT, the actual MICs based on the viable bacterial counts were determined in experiments with three pansusceptible strains (H37Rv, Atencio, Erdman). In each experiment two drugs were tested: RMP and RPT. Similar extensive work was done previously with RMP (7), and inclusion of this drug in the current experiment provided a control for consistency. The results of these experiments are presented in Table 1 . The actual MICs of both drugs determined by performing viable counts were only twofold higher than or equal to the MICs determined radiometrically for the same cultures.

Drug-resistant mutants. Laboratory strains with monoresistance to rifamycins were developed by cultivating each of the three pansusceptible strains mentioned above on 7H11 agar plates that contained $8 \mu \mathrm{g}$ of RPT or RMP per ml and that were inoculated with a heavy bacterial suspension of about $10^{10} \mathrm{CFU} / \mathrm{ml}$. From a few single colonies grown under these conditions, we subcultivated mutant substrains that were resistant to both rifamycins at concentrations above $8.0 \mu \mathrm{g} / \mathrm{ml}$. Each of these substrains represented a pure $100 \%$ resistant culture as determined by the agar proportion method. Both 
TABLE 1. Comparison of MICs determined in $7 \mathrm{H} 12$ broth radiometrically (GI) and by the viable count (CFU per milliliter) $\operatorname{method}^{a}$

\begin{tabular}{cccccc}
\hline \multirow{2}{*}{ Strain } & \multicolumn{2}{c}{ RMP MIC $(\mu \mathrm{g} / \mathrm{ml})$} & & \multicolumn{2}{c}{ RPT MIC $(\mu \mathrm{g} / \mathrm{ml})$} \\
\cline { 2 - 3 } \cline { 5 - 6 } & $\begin{array}{c}\text { Radiometric } \\
\text { method }\end{array}$ & $\begin{array}{c}\text { Viable count } \\
\text { method }\end{array}$ & & $\begin{array}{c}\text { Radiometric } \\
\text { method }\end{array}$ & $\begin{array}{c}\text { Viable count } \\
\text { method }\end{array}$ \\
\hline H37Rv & 0.06 & 0.06 & & 0.03 & 0.06 \\
Atencio & 0.06 & 0.12 & & 0.03 & 0.06 \\
Erdman & 0.06 & 0.12 & & 0.03 & 0.06 \\
\hline
\end{tabular}

${ }^{a}$ The data represent the results of three experiments performed with each strain.

types of these single-colony clones derived by selection with RMP and RPT were equally resistant to both drugs. One of these mutant clones derived from strain H37Rv in the presence of RPT was selected as a potential RPT-resistant QC strain, along with the original $\mathrm{H} 37 \mathrm{Rv}$ strain serving as a susceptible QC strain.

QC strains. Current standards of the Centers for Disease Control and Prevention, the Council of American Pathologists, and the National Committee for Clinical Laboratory Standards require only one QC strain (pansusceptible strain $\mathrm{H} 37 \mathrm{Rv}$ ) to be included with each batch of isolates tested in clinical laboratories. Nevertheless, for a test with a new antituberculosis agent (RPT), we felt that it was necessary to have two QC strains: one pansusceptible and one with monoresistance to RPT (strain H37RPT-R). Therefore, the results obtained with two strains, ATCC 27294 (pansusceptible) and ATCC 700457 (with monoresistance to RPT), were tested for their reproducibilities to justify their subsequent use as QC strains. Each experiment included simultaneous determination of the MICs of RPT and RMP by both the agar and the broth dilution methods. Such experiments for each of the two QC strains were repeated 10 times.

The results of 20 experiments with the susceptible and resistant QC strains are presented in Table 2. In 10 experiments with the susceptible strain in the BACTEC system, the results were identical, with no variation between the experiments: the MICs of RMP were always $0.12 \mu \mathrm{g} / \mathrm{ml}$, and the MICs of RPT were $0.03 \mu \mathrm{g} / \mathrm{ml}$ or less. Some variations were identified in the agar dilution test with both drugs, but the MICs of either drug usually did not exceed $0.12 \mu \mathrm{g} / \mathrm{ml}$, and the MIC of RMP was $0.5 \mu \mathrm{g} / \mathrm{ml}$ in only one experiment.

The actual MICs of RPT and RMP for the resistant QC strain were $32.0 \mu \mathrm{g} / \mathrm{ml}$, but in the reproducibility study we limited for practical purposes the range of concentrations to $8.0 \mu \mathrm{g} / \mathrm{ml}$. Ten experiments with the resistant QC strain had identical results: the highest concentration used in these reproducibility experiments, $8.0 \mu \mathrm{g}$ of either RMP or RPT per $\mathrm{ml}$, did not inhibit growth in either the broth or the agar

TABLE 2. Reproducibility of the agar and broth dilution methods: MICs of RPT and RMP for two QC strains, susceptible strain H37Rv and strain H37RPT-R with monoresistance to rifamycins

\begin{tabular}{|c|c|c|c|c|c|}
\hline \multirow{2}{*}{ Strain } & \multirow{2}{*}{ No. of expts } & \multicolumn{2}{|c|}{$\begin{array}{l}\text { MIC }(\mu \mathrm{g} / \mathrm{ml}) \text { by } \\
\text { BACTEC method }\end{array}$} & \multicolumn{2}{|c|}{$\begin{array}{l}\text { MIC }(\mu \mathrm{g} / \mathrm{ml}) \text { by } \\
\text { agar plate method }\end{array}$} \\
\hline & & RMP & RPT & RMP & $\overline{\mathrm{RPT}}$ \\
\hline $\mathrm{H} 37 \mathrm{Rv}$ & 7 & 0.12 & $\leq 0.03$ & 0.12 & $\leq 0.03$ \\
\hline $\mathrm{H} 37 \mathrm{Rv}$ & 2 & 0.12 & $\leq 0.03$ & 0.12 & 0.12 \\
\hline $\mathrm{H} 37 \mathrm{Rv}$ & 1 & 0.12 & $\leq 0.03$ & 0.5 & 0.12 \\
\hline H37RPT-R & 10 & $>8.0$ & $>8.0$ & $>8.0$ & $>8.0$ \\
\hline
\end{tabular}

system. These data indicated very substantial differences in the MICs for the susceptible and resistant strains, confirming their suitability as QC strains.

Sensitivity of the rapid BACTEC method for detection of low-level resistance to RPT and RMP. The important advantage of the BACTEC indirect susceptibility test over the 7H10 and 7H11 agar proportion method is that the results can be obtained within less than 10 days instead of 3 weeks. Nevertheless, it is known from clinical experience, including our experience at the National Jewish Mycobacteriology Laboratory, that for a relatively small percentage of isolates the BACTEC method may not detect the low level of resistance to RMP and other conventional drugs at the early stages of emerging resistance. With the introduction of the new rifamycin, we conducted a study to evaluate the actual sensitivity of the BACTEC method for determination of this low level of resistance to either RPT or RMP. For this purpose, we prepared, using three strains (H37Rv, Erdman, Atencio), artificial mixtures containing 1,10 , and $50 \%$ of their rifamycin-resistant mutants mentioned above and tested these mixtures by both methods along with fully susceptible original strains and $100 \%$ resistant mutants. Table 3 indicates the actual proportions of resistant bacteria in the bacterial population (expressed as percentages) detected in these cultures on agar plates containing various drug concentrations, as well as the MICs derived from the results of tests on agar plates and in the BACTEC system. These experiments showed that resistance to either RPT or RMP may not be detected by the BACTEC method if the culture contained less than $10 \%$ resistant bacteria. This phenomenon explains the discrepancies between the agar proportion and BACTEC methods sometimes observed previously for RMP in clinical laboratories. This deficiency of the BACTEC method may not be of great practical importance, since the cultures consisting of clinical isolates identified as "resistant" rarely contain fewer than $10 \%$ resistant bacteria. For example, there were no discrepancies between the agar proportion and BACTEC methods in detecting resistance to RMP or RPT in the experiments with 33 rifamycin-resistant clinical isolates described below.

MICs of RPT for pansusceptible strains. The MICs of RPT for eight laboratory strains were determined by the agar and broth dilution methods; these included repeat tests for three of the strains for which MIC data are presented in Table 1. It should be mentioned that the radiometric MICs in these experiments were only 1 dilution higher $(0.06$ versus $0.03 \mu \mathrm{g} / \mathrm{ml})$ for two strains and the same $(0.03 \mu \mathrm{g} / \mathrm{ml})$ for one of those tested in the preliminary experiments (Table 1$)$. The results of these experiments showed that the MICs of RPT by either method did not exceed $0.12 \mu \mathrm{g} / \mathrm{ml}$ (Table 4). Among the large number of pansusceptible isolates obtained from patients who had negative cultures following 60 days of therapy, the RPT MICs were also in the range of 0.03 to $0.12 \mu \mathrm{g} / \mathrm{ml}$ for 93 of 95 isolates, and for only 2 isolates the MICs were $0.25 \mu \mathrm{g} / \mathrm{ml}$ (Table 5). These data indicated that $0.5 \mu \mathrm{g}$ of RPT per ml could be considered a breakpoint for susceptibility.

MICs of RPT and RMP for resistant strains. Thirty-three clinical $M$. tuberculosis isolates that were reported in our clinical laboratory to be resistant to RMP by the conventional agar proportion method were included in this study. The MICs of both RPT and RMP were determined by two methods: on $7 \mathrm{H} 10$ and $7 \mathrm{H} 11$ agar plates and radiometrically in $7 \mathrm{H} 12$ BACTEC broth. The results of these experiments are presented in Table 6. In the agar plate experiments, the MICs of RPT were $8.0 \mu \mathrm{g} / \mathrm{ml}$ or greater than $8.0 \mu \mathrm{g} / \mathrm{ml}$ for 32 of 33 isolates and $4.0 \mu \mathrm{g} / \mathrm{ml}$ for only 1 of them. The MICs of RMP were $4.0 \mu \mathrm{g} / \mathrm{ml}$ for only 3 isolates, and for 30 isolates the RMP 
TABLE 3. Evaluation of sensitivity of BACTEC broth method for detection of resistance to RMP and RPT in strains containing small proportions of resistant bacteria

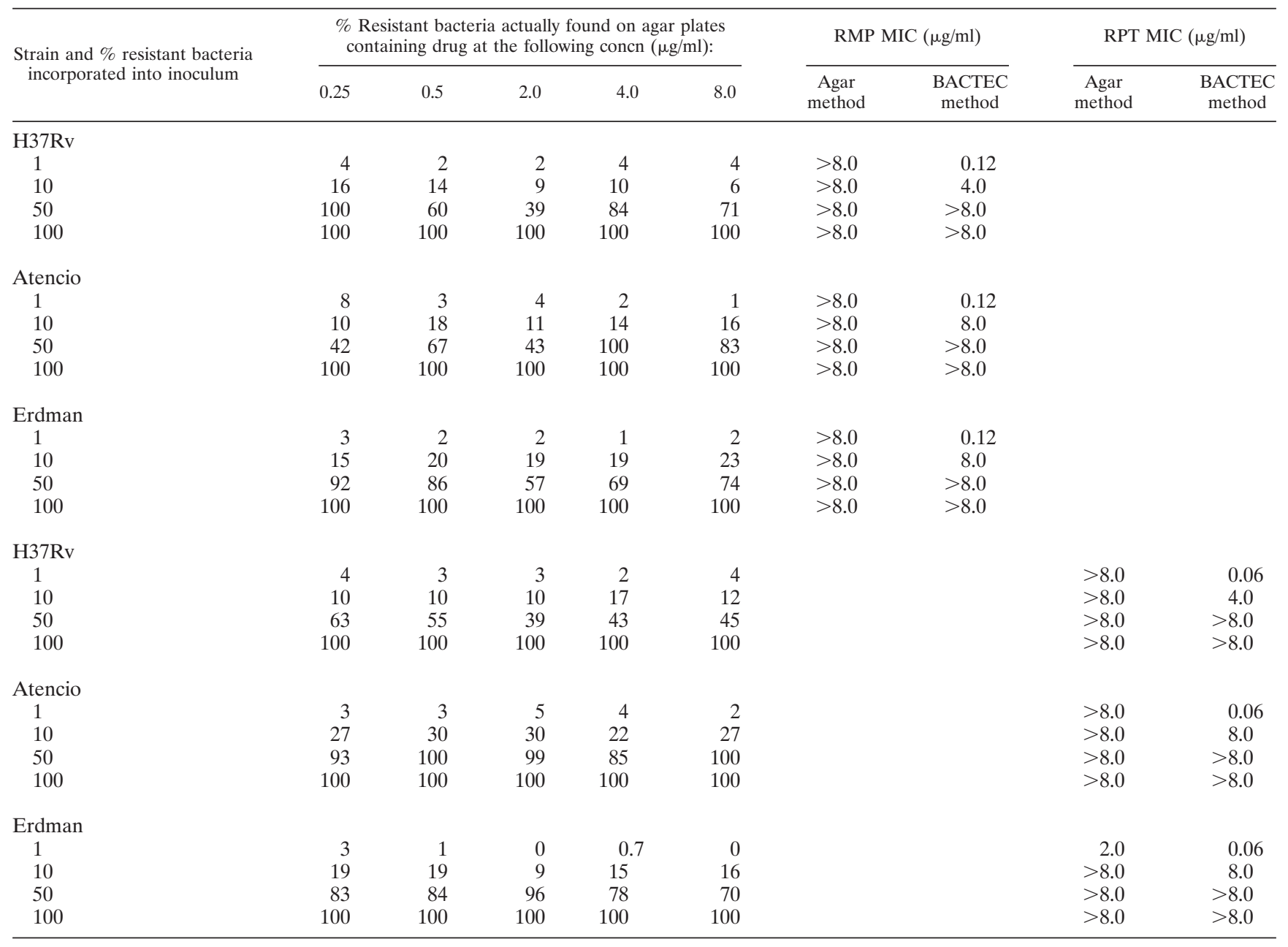

MICs were $\geq 8.0 \mu \mathrm{g} / \mathrm{ml}$. Identical results were obtained on $7 \mathrm{H} 10$ and $7 \mathrm{H} 11$ agar plates. In the BACTEC system the MICs of both RMP and RPT for all 33 isolates were $8.0 \mu \mathrm{g} / \mathrm{ml}$ or greater. These experiments confirmed full cross-resistance between RMP and RPT and that the RPT concentration of 0.5 $\mu \mathrm{g} / \mathrm{ml}$ suggested above as the breakpoint for susceptibility would not inhibit the growth of the rifamycin-resistant clinical isolates.

TABLE 4. MICs of RPT for eight pansusceptible M. tuberculosis strains determined on agar plates and in BACTEC broth

\begin{tabular}{lcc}
\hline \multirow{2}{*}{ Strain } & \multicolumn{2}{c}{ RPT MIC $(\mu \mathrm{g} / \mathrm{ml})$} \\
\cline { 2 - 3 } & Agar method & BACTEC method \\
\hline H37Rv & $\leq 0.03$ & 0.06 \\
Erdman & $\leq 0.03$ & 0.06 \\
Atencio & $\leq 0.03$ & 0.03 \\
12067 & $\leq 0.03$ & 0.06 \\
12060 & 0.06 & 0.12 \\
12066 & $\leq 0.03$ & 0.12 \\
12064 & $\leq 0.03$ & 0.06 \\
12058 & 0.25 & 0.12 \\
\hline
\end{tabular}

\section{DISCUSSION}

A critical concentration of $0.5 \mu \mathrm{g}$ of RPT per ml was developed for tests of the susceptibility of clinical M. tuberculosis isolates by two methods: the $7 \mathrm{H} 10$ and $7 \mathrm{H} 11$ agar proportion method and a radiometric method in BACTEC 7H12 broth (in $12 \mathrm{~B}$ vials). Two QC strains were evaluated: one strain that was susceptible to RPT and one strain that had monoresistance to RPT. We would suggest that with the introduction of the

TABLE 5. Distribution of 95 pansusceptible M. tuberculosis isolates obtained from 52 new patients by determination of MICs of RPT by the broth method

\begin{tabular}{cccccr}
\hline \multirow{2}{*}{ MIC $(\mu \mathrm{g} / \mathrm{ml})$} & \multicolumn{4}{c}{ No. of isolates obtained at the following times: } & \\
\cline { 2 - 5 } & $\begin{array}{c}\text { Before } \\
\text { therapy }\end{array}$ & $\begin{array}{c}\text { At } 30 \\
\text { days }\end{array}$ & $\begin{array}{c}\text { At } 60 \\
\text { days }\end{array}$ & $\begin{array}{c}\text { At } 90 \\
\text { days }\end{array}$ & Total \\
\hline$\leq 0.12$ & 50 & 28 & 15 & 0 & 93 \\
0.25 & 2 & 0 & 0 & 0 & 2 \\
0.5 & 0 & 0 & 0 & 0 & 0 \\
1.0 & 0 & 0 & 0 & 0 & 0 \\
Total & 52 & 28 & 15 & 0 & 95 \\
\hline
\end{tabular}


TABLE 6. Distribution of 33 drug-resistant $M$. tuberculosis isolates by determination of MICs of RPT and RMP

\begin{tabular}{lrrrrr}
\hline $\begin{array}{c}\text { Culture medium and RMP } \\
\text { MIC }(\mu \mathrm{g} / \mathrm{ml})\end{array}$ & \multicolumn{4}{c}{$\begin{array}{c}\text { No. of isolates for which the } \\
\text { RPT MIC }(\mu \mathrm{g} / \mathrm{ml}) \text { was as } \\
\text { follows: }\end{array}$} & Total \\
\cline { 2 - 4 } & 2.0 & 4.0 & 8.0 & $>8.0$ & \\
\hline 7 H10 and 7H11 agar plates & & & & & \\
2.0 & 0 & 0 & 0 & 0 & 0 \\
4.0 & 0 & 0 & 1 & 2 & 3 \\
8.0 & 0 & 1 & 0 & 1 & 2 \\
$>8.0$ & 0 & 0 & 3 & 25 & 28 \\
Total & 0 & 1 & 4 & 28 & 33 \\
BACTEC 7H12 broth & & & & & \\
4.0 & & 0 & 0 & 0 & 0 \\
8.0 & & 0 & 6 & 0 & 6 \\
$>8.0$ & & 0 & 5 & 22 & 27 \\
Total & & 0 & 11 & 22 & 33 \\
\hline
\end{tabular}

susceptibility test for this new drug in clinical laboratories both QC strains be used and, subsequently, that only the susceptible QC strain alone be used as required.

The proposal of the critical concentration of $0.5 \mu \mathrm{g} / \mathrm{ml}$ is based on determination of the MICs of RPT for 95 pansusceptible and 33 rifamycin-resistant isolates. The MICs for susceptible strains ranged from 0.03 to $0.12 \mu \mathrm{g} / \mathrm{ml}$ for most strains and were $0.25 \mu \mathrm{g} / \mathrm{ml}$ for only two of them. The MICs for most resistant strains were $8.0 \mu \mathrm{g} / \mathrm{ml}$ or greater, and the MIC was 4.0 $\mu \mathrm{g} / \mathrm{ml}$ for only one isolate. In other words, the concentration of $0.5 \mu \mathrm{g} / \mathrm{ml}$ was greater than the highest MIC of this drug in tests with pansusceptible isolates and it was substantially lower than the lowest MIC found in tests with the rifamycin-resistant isolates. Therefore, the concentration of $0.5 \mu \mathrm{g} / \mathrm{ml}$ served well in making a clear distinction between susceptible and resistant isolates by both methods. Nevertheless, it is well known from the experience in clinical laboratories that in some cases the drug susceptibility test in the BACTEC system with conventional drugs, particularly with RMP, may not detect resistance, causing a discrepancy with the result of a test performed on solid medium. We speculated that such a discrepancy may have been related to the isolates with low levels of drug resistance. For some drugs, for example, isoniazid, differences in the level of resistance may reflect the presence of mutants with different genetics on the basis of their low and high levels of resistance. Although the stepwise development of resistance of $M$. tuberculosis to rifamycins cannot be excluded, the fact that its mechanism of resistance to RMP and RPT is limited to the genetic alterations in an 81-bp region of the $r p o B$ gene may suggest that the differences in the degree of resistance to these drugs most likely reflects the differences in the proportions of resistant bacteria in a culture of the isolate. Practically, from among all rifamycin-resistant strains isolated in our laboratory (including 33 strains used for this study), we could not find a culture which would have a low level of resistance that was not related to the low proportion of resistant bacteria in the population. Therefore, to investigate the reason for relatively rare false-susceptible test results in the BACTEC system compared with the rate of occurrence of such results by the agar proportion method, we tested cultures of strains containing various proportions of rifamycin-resistant bacteria. We found that the test in the BACTEC system inconsistently detected low levels of resistance to either RMP or RPT in cultures in which the proportions of resistant bacteria were less than $10 \%$. It may not have great practical importance, since clinical isolates with such low levels of resistance are quite rare, probably because in patients the emerging resistance to rifamycins is usually progressing very rapidly. Nevertheless, this low probability of false-susceptible results should be taken into account when the BACTEC susceptibility test is the method of choice. This does not diminish the advantage of this method as a rapid indirect susceptibility test with a turnaround time of less than 10 days and as a means of obtaining reliable results for the vast majority of $M$. tuberculosis isolates. On the other hand, the indirect test by the agar proportion method, with a turnaround time of 3 to 4 weeks, has other advantages that must be taken into account: it is less expensive, it is more accurate in detecting low levels of resistance, and it provides the data on the actual proportion of resistant bacteria in the population. Beside this, the agar proportion method can be used as a direct test for smear-positive specimens, in which case the total turnaround time from the moment that the raw sputum sample is obtained can be almost the same as that for isolation plus an indirect test in the BACTEC system. The most rational approach is probably the use of a combination of both techniques in laboratory protocols, depending on the patient's condition, the type of specimens to be tested, laboratory capabilities, etc.

\section{ACKNOWLEDGMENTS}

This study was supported in part by Public Health Services grant U19-AI40972 from the National Institutes of Allergy and Infectious Diseases and in part by a grant from Hoescht Marion Roussel, Inc.

\section{REFERENCES}

1. Arioli, V., M. Berti, G. Carniti, E. Randisi, E. Rossi, and R. Scotti. 1981. Antibacterial activity of DL473, a new semisynthetic rifamycin derivative. J. Antibiot. (Tokyo) 34:1026-1029.

2. Dhillon, J., J. M. Dickinson, A. J. Gay, T. K. Ng, and D. A. Mitchison. 1989. Activity of two long lasting rifamycins, rifapentine and FCE22807, in experimental murine tuberculosis. Tubercle Lung Dis. 73:116-123.

3. Dhillon, J., and D. A. Mitchison. 1992. Activity in vitro of rifabutin, FCE 22807, rifapentine, and rifampin against Mycobacterium microti and $M$. tuberculosis and their penetration into mouse peritoneal macrophages. Am. Rev. Respir. Dis. 145:212-214.

4. Dickinson, J. M., and D. A. Mitchison. 1987. In vitro properties of rifapentine (MDL473) relevant to its use in intermittent chemotherapy of tuberculosis. Tubercle 68:113-119.

5. Heifets, L. 1996. Drug susceptibility testing in mycobacteriology, p. 641-656. In L. Heifets (ed.), Clinics in laboratory medicine: clinical mycobacteriology, vol. 16. The W. B. Saunders Co., Philadelphia, Pa.

6. Heifets, L. B. 1991. Drug susceptibility tests in the management of chemotherapy of tuberculosis, p. 89-121. In L. B. Heifets (ed.), Drug susceptibility in the chemotherapy of mycobacterial infections. CRC Press, Inc., Boca Raton, Fla.

7. Heifets, L. B., P. J. Lindholm-Levy, and M. A. Flory. 1990. Bactericidal activity in vitro of various rifamycins against $M$. avium and $M$. tuberculosis. Am. Rev. Respir. Dis. 141:626-630.

8. Ji, B., C. Truffot-Pernot, C. Lacroix, M. C. Raviglione, R. J. O'Brien, P. Olliaro, G. Roscigno, and J. Grosset. 1991. Effectiveness of clarithromycin and minocycline alone and in combination against experimental Mycobacterium leprae infection in mice. Antimicrob. Agents Chemother. 35:579-581.

9. Mor, N., B. Simon, N. Mezo, and L. Heifets. 1995. Comparison of the activities of rifapentine and rifampin against Mycobacterium tuberculosis residing in human macrophages. Antimicrob. Agents Chemother. 39:20732077.

10. Pascual, A., D. Tsukayama, J. Kovarik, G. Gekker, and P. Peterson. 1987. Uptake and activity of rifapentine in human peritoneal macrophages and polymorphonuclear leukocytes. Eur. J. Clin. Microbiol. 6:152-157.

11. Truffot-Pernot, C. H., J. Grosset, R. Bismuth, and H. L. Lecoeur. 1983. Activité de la rifapicine administrée de maniére intermittente et de la cyclopentyl rifamycine (ou DL473) sur la tuberculose expérimentale de la souris. Rev. Fr. Mal. Respir. 11:875-882.

12. Tsukamura, M., S. Mizuno, and H. Toyama. 1986. In vitro antimycobacterial activity of rifapentine (comparison with rifampicin). Kekkaku 61:633-639.

13. Yates, M. D., and C. H. Collins. 1982. Comparison of the sensitivity of mycobacteria to the cyclopentyl rifamycin DL473 and rifamycin. J. Antimicrob. Chemother. 10:147-150. 